\title{
Cryoprotective Effects of Cycloinulohexaose on Freezing and Freeze-Drying of Liposomes
}

\author{
Kazuhiko OZAKI ${ }^{*, a}$ and Masahiro HAYASHI ${ }^{b}$ \\ Pharmaceuticals Development Laboratory, Mitsubishi Chemical Corporation, ${ }^{a} 14$ Sunayama, Hasaki-machi, \\ Kashima-gun, Ibaraki 314-02, Japan and Faculty of Pharmaceutical Sciences, Science University of Tokyo, 12 \\ Ichigaya, Funagawara-machi, Shinjuku-ku, Tokyo 162, Japan. Received May 9, 1996; accepted July 31, 1996
}

Cycloinulohexaose (CF-6) maintained the membrane structure of the liposomes during freeze-thawing, and thus CF-6 was very effective in inhibiting both drug leakage from liposomes and the size change of liposomes during freezing and freeze-drying. As the ratio of CF-6 to lipid increased from 1 to 5, calcein retention in the liposomes increased from $70 \%$ up to $85 \%$ and a maximum retention was obtained at a ratio of more than 2 . The addition of glycerol enhanced the above cryoprotective effect of CF-6 and improved calcein retention up to $90 \%$. Neither detectable leakage of calcein nor particle size change was observed after the storage of liposomes freeze-dried with CF-6 for 6 months at 4 and $25^{\circ} \mathrm{C}$.

Key words liposome; cycloinulohexaose; freeze; freeze-dry

Recently, liposomal formulations for many kinds of drugs have been studied for drug delivery systems, ${ }^{1,2}$ but the instability of liposomes remains the biggest problem for their commercialization. The freezing and freeze-drying of liposomes with cryoprotectants is one of the most promising ways to solve this problem. Saccharides, especially di-saccharides such as trehalose and maltose, have been extensively investigated as cryoprotectants of liposomes. ${ }^{3,4)}$ It is well known that these saccharides have two kinds of effects. One is to prevent the mechanical breaking of the membrane caused by ice crystals and fusion during freezing. The other is to prevent the fusion of the membrane during drying or rehydration. One of the problems in using disaccharides as a cryoprotectant is their low collapse temperature. Samples must be kept below their collapse temperatures during the primary drying of freeze-drying. And glucoside chains longer than tri-glucoside show less cryoprotective effects than glucoside chains of mono, di and tri-glucosides. ${ }^{5)}$ Therefore, cryoprotectants which have high collapse temperatures are needed to prepare freeze-dried liposomes of good quality and stability which are relatively easy to reproduce.

In this paper, we report that cycloinulohexaose (CF-6) has the ability to act as a cryoprotectant during the freezing and freeze-drying liposomes. CF-6, which is produced from inulin, is a cyclohexaose of $\beta$-D-fructofranose consisting of $\beta(2 \rightarrow 1)$-linked oligofructose chains (Fig-1). ${ }^{6}$ The production of CF-6 is catalyzed by the action of extracellular cycloinulo-oligosaccharide fructanotransferase. It was also shown that the collapse temperature of CF-6 solution was $-25^{\circ} \mathrm{C}$, which is slightly higher than that of mono- $\left(-48^{\circ} \mathrm{C}\right)$ and di-saccharides $\left(-32^{\circ} \mathrm{C}\right)$. If cryoprotectants with a high collapse temperature could be used for the freeze-drying of liposomes, the temperature of liposomes during the drying process could be maintained at that temperature and it must be possible to reduce the time for the drying process. It is thus suggested that CF-6 has a big advantage as a cryoprotectant for the freezing and freeze-drying of liposomes.

\section{Experimental}

Materials CF-6 was synthesized at the Research Center, Mitsubishi Chemical Corp. (Yokohama, Japan). ${ }^{7)}$ The purity of CF-6 was greater than $97 \%$. L- $\alpha$-Dipalmitoylphosphatidylcholine (DPPC) was purchased from Nippon Oil and Fats Co., Ltd. (Tokyo, Japan); egg phosphatidylcholine (EPC) from Asahi Chemical Ind. Co., Ltd. (Tokyo, Japan); cholesterol (Chol) from Sigma (U.S.A.); trehalose, saccharose, glucose and fructose from Wako Chemical Co., Ltd. (Tokyo, Japan); calcein from Dojindo (Kumamoto, Japan). Water was purified by deionization and distillation. Polycarbonate membrane filters were purchased from Caster (Cambridge, U.S.A.). The other chemicals used in this study were reagent grade or better.

Preparation of Liposomes Multilamellar vesicles (MLV) were prepared by Bangham's method. ${ }^{8)}$ The lipids were dissolved in chloroform in a round bottomed flask and were dried via rotary evaporation to make a thin film. The residual solvent was removed in the desiccator. Calcein solution $(95 \mathrm{~mm}, \mathrm{pH} 7.0)$ was added to the round bottomed flask, and the flask was shaken by a vortex mixer for about $10 \mathrm{~min}$ above $60^{\circ} \mathrm{C}$ to make the MLV liposomes. The osmotic

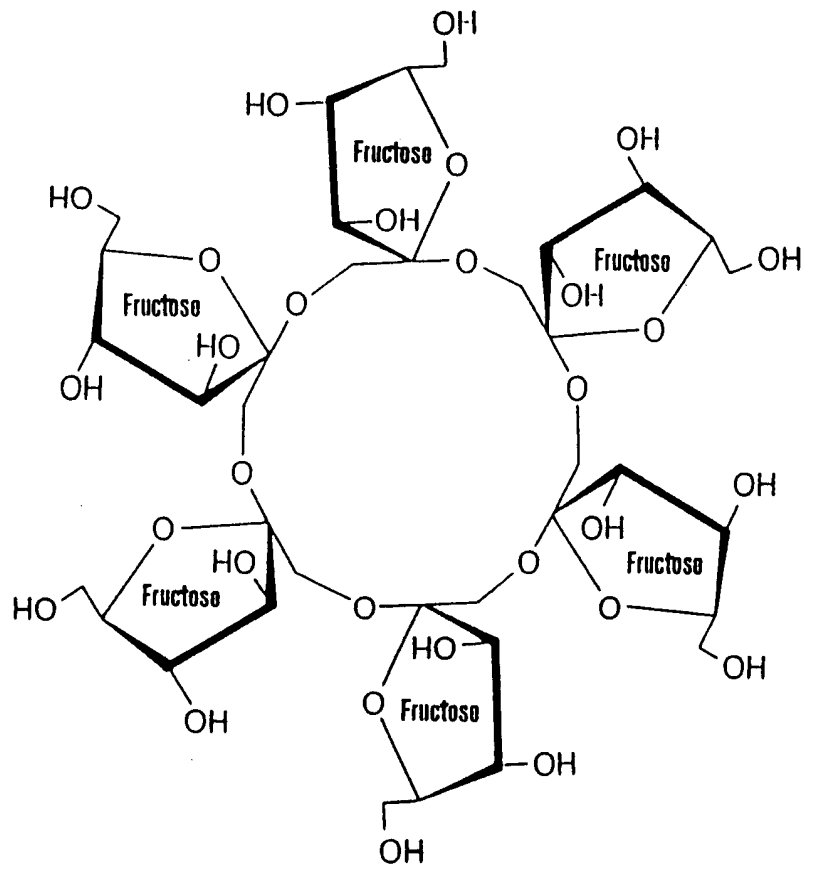

Fig. 1. The Structure of Cycloinulohexaose

Formula: $\mathrm{C}_{36} \mathrm{H}_{60} \mathrm{O}_{30} \cdot 3 \mathrm{H}_{2} \mathrm{O}$. Molecular weight: 1026.9 .

(C) 1996 Pharmaceutical Society of Japan 
pressure of the calcein solution was adjusted to that of the solution outside the liposomes, in which the cryoprotectants were added. To adjust the liposome size, the liposome solution was extruded through two polycarbonate membrane filters with $100 \mathrm{~nm}$ diameter pores at above $60^{\circ} \mathrm{C}$. The extrusion was repeated 10 times. Untrapped calcein was removed using a Sepharose-6FF column by using distilled water as an eluent. Then, the cryoprotectants were added to the liposomal solution to make it isotonic to the solution inside the liposomes, and finally the concentration of lipids was adjusted to $20 \mathrm{mg} / \mathrm{ml}$.

Particle Size The mean particle size of the liposomes was determined by a quasi-elastic laser light scattering measurement using a Coulter N4 (Coulter Electronics, Inc., U.S.A.).

Frozen Liposomes Two $\mathrm{ml}$ of the liposomal solution was placed into $10 \mathrm{ml}$ glass vials and the vials were sealed with rubber stoppers and aluminum caps. Then, the vials were frozen at $-80^{\circ} \mathrm{C}$ in a freezer. Liposomal size and calcein leakage of each of the samples were measured after thawing the liposomes to room temperature.

Freeze-Dried Liposomes Two $\mathrm{ml}$ of the liposomal solution was placed into $10 \mathrm{ml}$ glass vials. The vials were half sealed by rubber stoppers. The vials were then lyophilized using RL-100BSW (Kyowa Vacuum Engineering, Ltd., Japan). The liposomes were frozen to a final shelf temperature of $-40^{\circ} \mathrm{C}$ and vacuumed at a pressure of $0.05 \mathrm{mbar}$. Then, the shelf temperature was set at $-20^{\circ} \mathrm{C}$ for $20 \mathrm{~h}$, and thereafter its temperature was set at $20^{\circ} \mathrm{C}$ for $10 \mathrm{~h}$. Dried nitrogen gas was emitted into the drying chamber and the vials were sealed inside the drying chamber. Liposomal size and calcein leakage of each of the samples was measured after rehydration using $2 \mathrm{ml}$ of distilled water.

Retention of Calcein (\%) Leaked free calcein from each sample was separated from liposomes using a Sepharose-6FF column. Ten $\mu \mathrm{l}$ of $10 \%$ Triton X-100 solution was added to the liposomal fraction and the mixture was then heated at $60^{\circ} \mathrm{C}$. The amount of free calcein and the calcein in the liposomes was determined by fluorescence measurement using an RF-5000 (Shimadzu, Japan) at $490 \mathrm{~nm}$ for excitation and $520 \mathrm{~nm}$ for emission.

${ }^{1}$ H-NMR Spectra of Liposomes EPC was dissolved in chloroform and dried via rotary evaporation. Then, $\mathrm{D}_{2} \mathrm{O}$ with and without $10 \%$ CF-6 (w/w) were added to EPC film. MLV and sized liposomes were made by the method described above. All NMR samples were adjusted to $100 \mathrm{mg} / \mathrm{ml}$ (EPC). Homo gate decouple spectra were obtained using a pulse width of $45^{\circ}$ in a GSX-400 (JEOL, Japan).

Differential Seanning Calorimetry (DSC) Measurement Fifty mg of DPPC was dissolved in chloroform and dried via rotary evaporation.

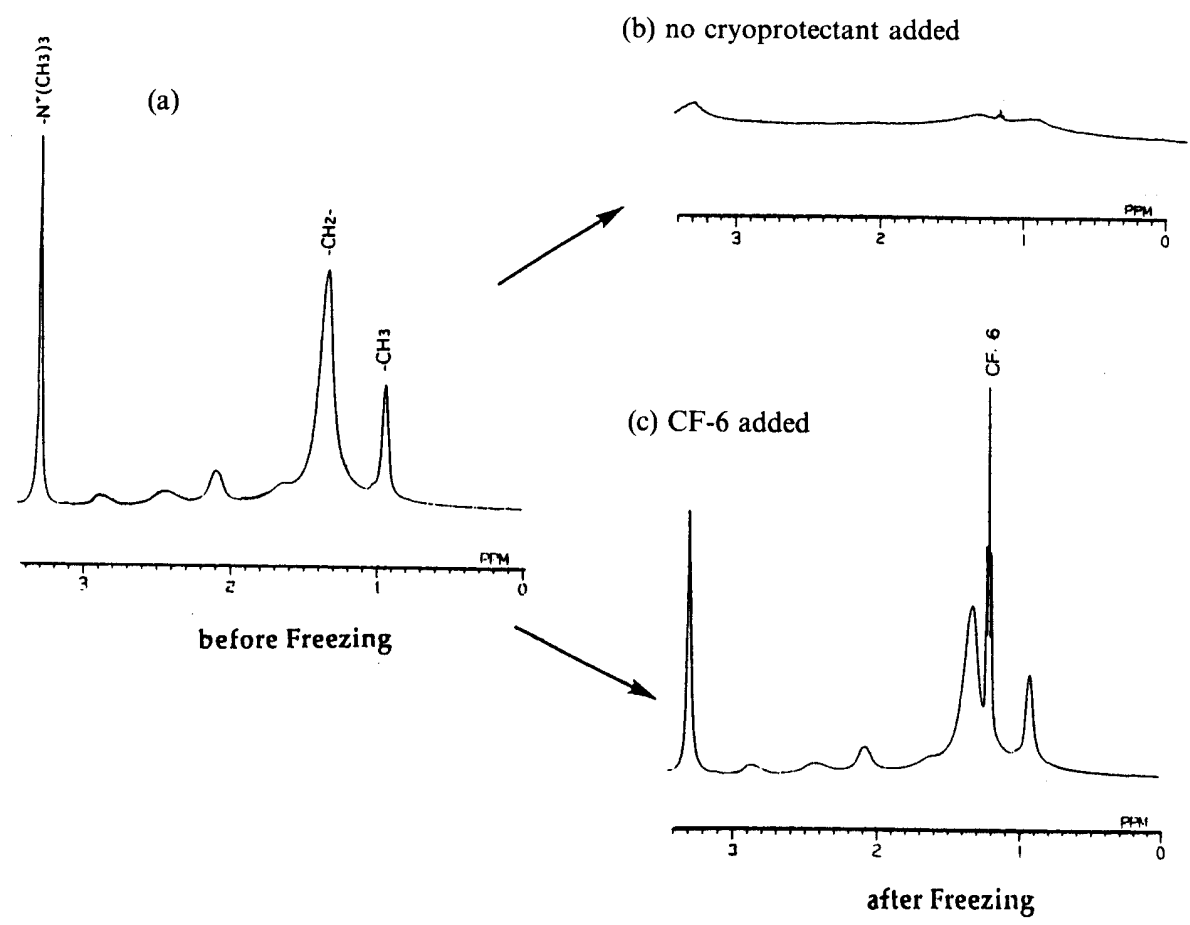

Fig. 2. The ${ }^{1} \mathrm{H}$-NMR Spectra of Liposomes in $\mathrm{D}_{2} \mathrm{O}$ Obtained after Freeze-Thawing

a) Before freezing. $b$ ) After freeze-thawing without any cryoprotectant. $c$ ) After freeze-thawing with CF-6.
Then, $100 \mathrm{mg}$ of saccharide in $1 \mathrm{~g}$ of distilled water was added. MLV and sized liposomes were made by the method described above. Aluminum DSC pans filled with $20 \mathrm{mg}$ of liposomal solutions were placed in $10 \mathrm{ml}$ vials. The samples were lyophilized by the freeze-dried procedure described above. After that, the aluminum pans were hermetically sealed. The thermograms were analyzed using a DSC $8230 \mathrm{C}$ (Rigaku, Japan). The first scan was performed from $-10^{\circ} \mathrm{C}$ to $100^{\circ} \mathrm{C}$ at a heating rate of $10^{\circ} \mathrm{C} / \mathrm{min}$. The second scan, after annealing the samples at $100^{\circ} \mathrm{C}$ for $5 \mathrm{~min}$, was performed from $-10^{\circ} \mathrm{C}$ to $100^{\circ} \mathrm{C}$ at a heating rate of $10^{\circ} \mathrm{C} / \mathrm{min}$.

\section{Results}

Cryoprotective Effects of CF-6 on the Freezing of Liposomes A choline proton signal and a methylene and methyl proton signal from the acyl chain were observed on the ${ }^{1} \mathrm{H}$-NMR spectra of unilamellar liposomes in $\mathrm{D}_{2} \mathrm{O}$ solution (Fig. 2a). ${ }^{5}$ ) The half band width of the signals was increased after the freeze-thawing without any cryoprotectants, as shown in Fig. 2 b. On the other hand, these peaks were observed after the freeze-thawing process when CF- 6 was added (Fig. 2c). And, as shown in Tables 1 and 2 , the particle size and retention of calcein (\%) after freeze-thawing with CF- 6 remained unchanged from those prior to the treatment.

Cryoprotective Effects of CF-6 on the Freeze-Drying of Liposomes As shown in Tables 1 and 2, the size change of liposomes and the leakage of calcein during freezedrying were prevented by the addition of CF-6 at almost the same effectiveness as mono- and di-saccharides. In the case of liposomes composed of cholesterol and DPPC, the retention of calcein was decreased in proportion to an increase in cholesterol content (Fig. 3).

As shown in Fig. 4, the thermal analysis of DPPC liposomes freeze-dried with CF-6 was investigated. The gel-to-liquid crystalline phase transition temperature $\left(T_{\mathrm{m}}\right)$ of DPPC at the first scan was about $70^{\circ} \mathrm{C}$, whereas the $T_{\mathrm{m}}$ at the second scan was about $26^{\circ} \mathrm{C}$. The same behavior

(b) no cryoprotectant added 
Table 1. Mean Diameter of Liposomes (DPPC/Chol $=80 / 20$, mol Ratio) Following Freezing or Freeze-Drying with Various Cryoprotec$\operatorname{tants}^{a, b)}$

\begin{tabular}{lcc}
\hline \hline Cryoprotectant & After freezing & After freeze-drying \\
\hline CF-6 & $92.5 \pm 26 \mathrm{~nm}^{c}$ & $98.9 \pm 28 \mathrm{~nm}$ \\
Trehalose & $89.5 \pm 24 \mathrm{~nm}$ & $92.0 \pm 22 \mathrm{~nm}$ \\
Saccharose & $88.0 \pm 22 \mathrm{~nm}$ & $89.2 \pm 21 \mathrm{~nm}$ \\
Fructose & $88.5 \pm 24 \mathrm{~nm}$ & $94.7 \pm 25 \mathrm{~nm}$ \\
Glucose & $92.7 \pm 26 \mathrm{~nm}$ & $92.4 \pm 22 \mathrm{~nm}$ \\
No additive & $>3000 \mathrm{~nm}$ & $>3000 \mathrm{~nm}$
\end{tabular}

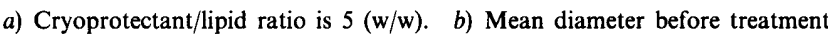
is $82.3 \pm 21 \mathrm{~nm}$. c) Mean diameter \pm S.D. (size distribution).

Table 2. Calcein Retention by Liposomes (DPPC/Chol $=80 / 20$, mol Ratio) Following Freezing or Freeze-Drying with Various Cryoprotectants $(n=3)^{a)}$

\begin{tabular}{lcc}
\hline Cryoprotectant & After freezing & After freeze-drying \\
\hline CF-6 & $98.5 \pm 0.1 \%$ & $84.8 \pm 3.9 \%$ \\
Treharose & $98.5 \pm 0.2 \%$ & $88.6 \pm 2.9 \%$ \\
Saccharose & $98.4 \pm 0.8 \%$ & $92.1 \pm 2.0 \%$ \\
Fructose & $98.4 \pm 0.9 \%$ & $84.9 \pm 5.3 \%$ \\
Glucose & $98.1 \pm 1.7 \%$ & $88.0 \pm 4.0 \%$ \\
No additive & $\fallingdotseq 0 \%{ }^{b)}$ & $\fallingdotseq 0 \%$ \\
\hline
\end{tabular}

a) Cryoprotectant/lipid ratio is $5(w / w) . ~ b)$ Could not pass through the gel filtration column.

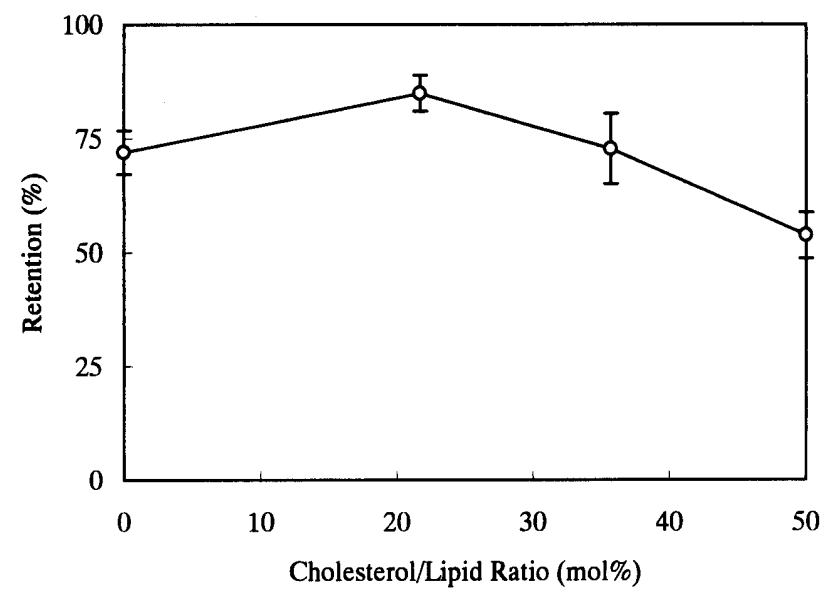

Fig. 3. Relationship between Cholesterol Content (mol\%) and Calcein Retention (Following Freeze-Drying and Rehydration)

CF-6 $(100 \mathrm{mg} / \mathrm{ml})$ was added to the outside of the liposomes $(n=3)$.

is exhibited by di-saccharides such as maltose. ${ }^{9,10)}$

Cryoprotective Effects of CF-6 in the Presence of Glycerol The cryoprotective effects of CF-6 were detected, but about $15 \%$ of calcein still leaked out during freezedrying. In order to reduce this leakage, the cryoprotective effect of CF-6 in the presence of glycerol was investigated. It is well known that glycerol prevents the mechanical damage of liposome membranes during the freeze-thawing step, but glycerol has no effect during the drying step. ${ }^{11)}$ Glycerol and CF-6 were added to the liposomal solution at final concentrations of $15 \mathrm{mg} / \mathrm{ml}$ and $100 \mathrm{mg} / \mathrm{ml}$, respectively. Calcein retention after the rehydration of freeze-dried liposomes in the presence of both glycerol and CF- 6 was improved to about $90 \%$. In the case of trehalose, the addition of glycerol yielded a lower degree

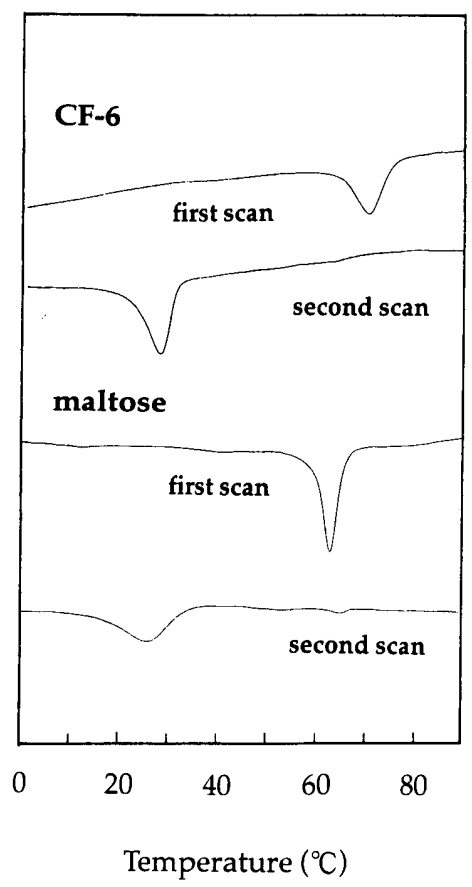

Fig. 4. First and Second DSC Thermograms of Lyophilized DPPC Liposomes with CF-6 and Maltose $(100 \mathrm{mg} / \mathrm{ml})$

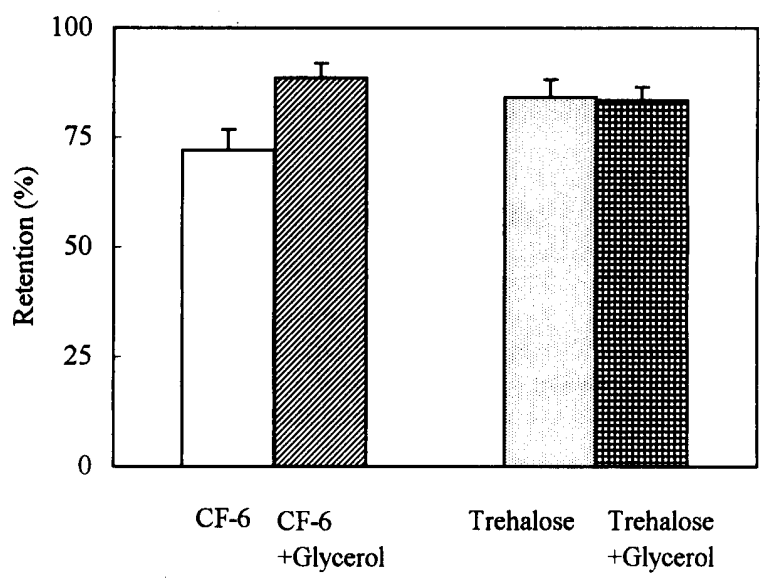

Fig. 5. Reinforcement of the Effect on Calcein Retention by the Addition of Glycerol

Glycerol $(15 \mathrm{mg} / \mathrm{ml})$ was added to the outside solution of DPPC liposomes with CF-6 or trehalose $(100 \mathrm{mg} / \mathrm{ml})(n=3)$.

of effectiveness, as shown in Fig 5. The same result was obtained by Harrigan et al. ${ }^{12)}$ for EPC liposomes during dehydration.

The Effects of Varying Amounts of CF-6 Added to a Set Amount of lipid The effects of the ratio of CF-6 to lipids on the stability of freeze-dried liposomes was investigated (Fig. 6). As the ratio increased from 1 to 2, the calcein retention increased from $70 \%$ up to $85 \%$, and this increase was saturated above the ratio of $2(1.4 / 1$ on the molar ratio).

Long-Term Stability of Freeze-Dried Liposomes with CF-6 Freeze-dried liposomes with CF-6 were stored for 6 months at both $4{ }^{\circ} \mathrm{C}$ and $25^{\circ} \mathrm{C}$. As shown in Fig 7, no noticeable leakage of calcein or change in particle size was observed after 24 weeks at both $4{ }^{\circ} \mathrm{C}$ and $25^{\circ} \mathrm{C}$. It was thus indicated that freeze-drying with $\mathrm{CF}-6$ was one of the promising means of achieving the long-term stability of liposomes. 


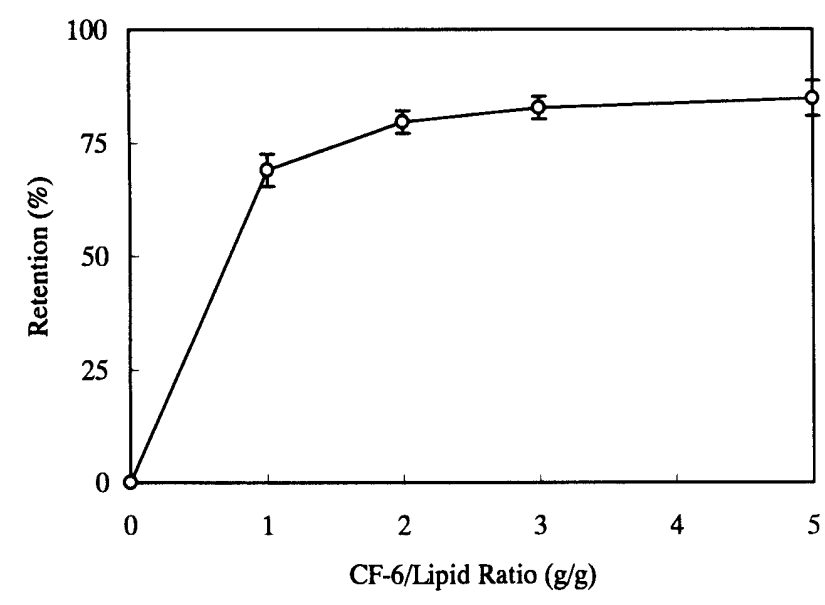

Fig. 6. Relationship between the CF-6/Lipid Ratio and Calcein Retention (Following Freeze-Drying and Rehydration)

CF-6 was added to the outside of the liposomes (DPPC/Chol $=80 / 20$, molar ratio, $n=3$ ).

a)

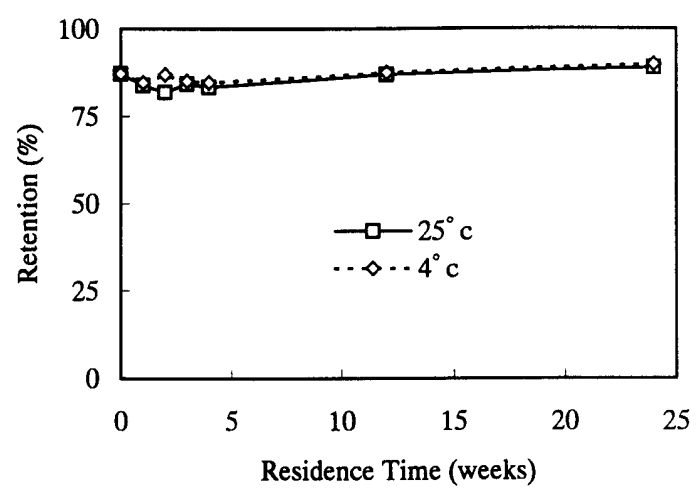

b)

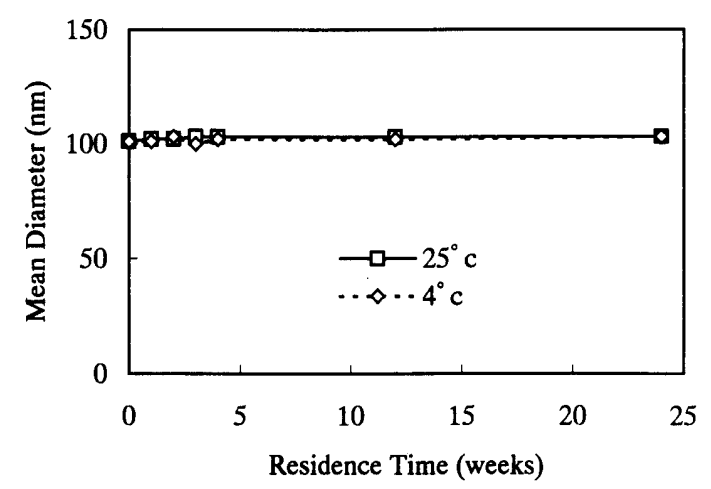

Fig. 7. Relationship between Storage Time and a) Calcein Retention and b) Mean Diameter of Liposomes (Following Freeze-Drying and Rehydration)

Freeze-dried liposomes were stored at both $4{ }^{\circ} \mathrm{C}$ and $25^{\circ} \mathrm{C}$. CF-6 $(100 \mathrm{mg} / \mathrm{ml})$ was added to the outside of liposomes (DPPC/Chol $=80 / 20$, molar ratio).

\section{Discussion}

The results of Fig. 2 suggest that the mutual interaction of the neighboring acyl chain is stronger after freezethawing without CF-6. This result suggests that unilamellar liposomes were fused and became large multilamellar liposomes during freeze-thawing without any cryoprotectants, and these change were prevented during freeze-thawing with CF-6 at the same level of effectiveness as with mono- and di-saccharides. ${ }^{5)}$ These results were inconsistent with the results by Miyajima et $a l .{ }^{5)}$ which showed that glucoside chains longer than tri-glucoside show smaller cryoprotective effects. One of the reasons for this difference may be a difference in experimental conditions. In their experiment, EPC sonicated liposomes were used. Also the $T_{\mathrm{m}}$ of hydrated EPC was lower than room temperature (about -15$-17^{\circ} \mathrm{C}$ ). So, during the rehydration process, EPC liposomes must pass through their $T_{\mathrm{m}}$. But in the case of DPPC liposomes, they do not pass through their $T_{\mathrm{m}}$ during the rehydration process. Liposome membranes sustained damage if they passed through their $T_{\mathrm{m}}$. However, CF-6 was considered to have a beneficial cryoprotective effect on liposomal membranes during the freeze-thawing process.

As shown in Fig. 3, the same results were obtained by Tanaka et al. ${ }^{13)}$ for EPC liposomes with trehalose. They reported that destabilization was caused by the phase separation of lipids and cholesterol. Moreover, Lentz et al. ${ }^{14)}$ noted that a phase separation was observed in DPPC-cholesterol systems.

As shown in Fig. 4, one of the mechanisms of this cryoprotection by CF-6 was due to an increase in lipid membrane fluidity in a dry state, which prevents leakage during the rehydration process. However, the $T_{\mathrm{m}}$ in the presence of CF-6 at the first scan was a little higher than that of maltose. It was indicated that after the annealing process, the interactions between CF- 6 and DPPC became strong, in the same manner as maltose. Before annealing, these interactions were weaker than di-saccharides. As CF-6 is a somewhat more hydrophobic than di-saccharides, the interaction between CF- 6 and lipids may be a little difficult before annealing, resulting in a little lower retention of calcein in the presence of CF- 6 than with mono- and di-saccharides after freeze-drying.

As shown in Fig. 5, the effect of CF-6 in the presence of glycerol was different from the effect of trehalose in the presence of glycerol. Comparing the freeze-dried cakes of both, that of trehalose with glycerol was shrunk. This indicated that the cake collapsed during the drying process. In the case of trehalose with glycerol, the collapse temperature was about $-41^{\circ} \mathrm{C}$. And in case of CF-6 with glycerol, the collapse temperature was about $-36^{\circ} \mathrm{C}$. Because of these differences in collapse temperature, in case of trehalose, the cake was collapsed and the cryoprotective effects were not improved with glycerol under our experimented conditions. However, the reason for this improvement of CF- 6 with glycerol is not yet clear. Further study is required to determine the reason for this improvement.

Crowe et $a .^{15)}$ reported that in the case of trehalose, 1.8 times the amount $(\mathrm{w} / \mathrm{w})$ of trehalose to lipids is essential for the almost perfect prevention of leakage and size change of liposomes (a molar ratio of about 4/1). As shown in Fig. 6, although the amount ratio of CF-6 to lipid was almost the same as that of trehalose to lipid, the molar ratio of CF-6 to lipid was different from that of trehalose to lipid. However, the molar ratio of glucoside units to lipid was almost the same between CF-6 and 
trehalose, at 8.58 and 8 , respectively. This indicated that what is important for cryoprotection is the molar ratio of the glucoside unit in the saccharides to the lipid, but not the molar ratio of saccharide to lipid.

\section{Conclusion}

CF-6 has the ability to exert cryoprotective effects on liposomes during freezing and freeze-drying. Although the mechanism of the effects of CF-6 is not yet clear, it is indicated an increase in lipid membrane fluidity at a dry state might prevent leakage during the rehydration of the liposome in the same way as di-saccharides. The cryoprotective effects were increased by adding glycerol together with CF-6. These effects might be related to the molar ratio of the glucoside unit in oligosaccharides to lipids. These liposomes were stable over 6 months. In conclusion, CF- 6 is considered to be a very useful cryoprotectant of liposomes during freezing and freezedrying.

\section{References}

1) Bally M. B., Masin D., Nayar R., Cullis P. R., Mayer L. D., Cancer Chemother. Pharmacol., 34, 137-146 (1994).

2) Litzinger D. C., Buiting A. M. J., Rooijen N., Huang L., Biochim.
Biophys. Acta, 1190, 99-107 (1994).

3) Crowe J. H., Crowe L. M., Carpenter J. F., Rudolph A. S., Wistron C. A., Spargo B. J., Anchordoguy T. J., Biochim. Biophys. Acta, 947, 367-384 (1988).

4) Özer Y., Talsma H., Crommelin J. A., Hincal A. A., Acta Pharm. Technol., 34, 129-139 (1988).

5) Miyajima K., Tomita K., Nagaki M., Chem. Pharm. Bull., 34, 2689-2697 (1986).

6) Kawamura M., Uchiyama T., Kuramoto T., Tamura Y., Mizutani K., Calbohydr. Res., 192, 83-90 (1989).

7) Kushibe S., Sachida R., Morimoto Y., Biosci. Biotech. Biochem., 58, 1136 (1994).

8) Bangham A. D., Standish M.M., Watkins J. C., J. Mol. Biol., 13, 238-152 (1965).

9) Tanaka K., Takada T., Fujii K., Miyajima K., Chem. Pharm. Bull., 40, 1-5 (1992)

10) Cary Mobley W., Schreier H., J. Controlled Release, 31, 73-87 (1994).

11) Crowe L. M., Crowe J. H., "Liposome Techology," 2nd ed., ed. by Gregoriadis G., CRC, Boca Raton, 1992, pp. 229-252.

12) Harrigan P. R., Madden T. D., Cullis P. R., Chem. Phys. Lipids, 52, 139-149 (1990).

13) Tanaka K., Takada T., Fujii K., Miyajima K., Chem. Pharm. Bull., 39, 2653-2656 (1991)

14) Lentz B. R., Barrow D. A., Hoechli M., Biochemistry, 19, 1943-1954 (1980)

15) Crowe L. M., Crowe J. H., Rudolph A., Womersley C., Appel L., Arch Biochimica. Biophys., 242, 240-247 (1985). 